Journal of Case Reports 2020;10(2):86-89

\title{
A Rare Case of Myocardial Contusion
}

\author{
Sanjay Jaiswal, Ankur Verma, Anil Kumar Kadamba, Wasil Rasool Sheikh \\ Department of Emergency Medicine, Max Super Specialty Hospital, Patparganj, New Delhi, India.
}

\section{Corresponding Author: \\ Dr. Ankur Verma \\ Email: anksv25@gmail.com}

This is an Open Access article distributed under the terms of the Creative Commons Attribution License (creativecommons.org/ licenses/by/3.0).

Received : February 14, 2020

Accepted : April 1,2020

Published : April 30, 2020

\begin{abstract}
Background: Across the globe, trauma remains as one of the leading causes of morbidity and mortality. $25 \%$ of trauma mortalities can be attributed to blunt thoracic trauma. Myocardial contusions are perhaps the most uncommon injury reported after blunt thoracic trauma and no gold standard has been established to diagnose such patients. Patients with cardiac contusions have non-specific signs and symptoms which can easily be masked by other diagnoses like hemorrhagic shock, tension pneumothorax and tamponade. Case Report: A young male with blunt trauma presented to the emergency after a road traffic accident and succumbed due to myocardial contusions. Here we describe how we came to the diagnosis of myocardial contusions. Conclusion: It is imperative for emergency physicians to recognize myocardial contusions in blunt thoracic injuries early to avoid unfavorable outcomes.
\end{abstract}

Keywords: Myocardial Contusions, Pneumothorax, Thoracic Injuries, Traffic Accidents, Wounds.

\section{Introduction}

Across the globe, trauma remains as one of the leading causes of morbidity and mortality [1]. Out of all the traumatic fatalities, $25 \%$ can be attributed to blunt thoracic trauma [2]. Blunt cardiac injuries (BCI) can range from pericardial and valvular injuries to coronary injuries and myocardial contusions. Lack of gold standard diagnostics makes it difficult to pinpoint the exact type of BCI in trauma patients. Incidence of cardiac damage in blunt chest trauma has been reported as $8-75 \%$ [3], the wide range confirming the difficulty in diagnosis. Myocardial contusions are perhaps the most uncommon injury reported after blunt thoracic trauma, but definitive diagnostic tools for the same remains to be found. We present a case of a young male with blunt trauma causing myocardial contusions leading to mortality.

\section{Case Report}

A 37 year old previously healthy male was brought to the emergency department (ED) after being hit by a bus while jogging on the road. On primary survey, the patient was unconscious with Glasgow coma score of $11 / 15$, patent airway, pulse rate of $120 / \mathrm{min}$, blood pressure $140 / 90 \mathrm{mmHg}$, respiratory rate of 35/minute and oxygen saturation on room air of $90 \%$. The patient was applied a cervical collar and immediately intubated and mechanically ventilated. On secondary survey, the patient had two scalp lacerations of approximately $8 \mathrm{~cm}$ each which were stapled. Rest of the secondary examination was unremarkable.

The patient underwent the trauma series radiological investigations. Computed tomography (CT) of head and cervical spine, X-ray chest, $\mathrm{X}$-ray pelvis and FAST scan of abdomen were negative for any injuries. The patient was initially advised admission under the neurosurgical team. First electrocardiograph (ECG) of the patient had revealed sinus tachycardia. Initial laboratory findings were unremarkable Post investigations, the patient suddenly developed tachycardia and hypotension with a heart rate of $180 / \mathrm{min}$ and blood 
pressure of $80 / 60 \mathrm{mmHg}$. The patient was started on intravenous fluids, blood products and inotropic support. The blood pressure settled at 110/80 mmHg. Repeat ECG showed supra-ventricular tachycardia (SVT), which resolved to sinus tachycardia spontaneously. Repeat FAST scan did not reveal any positive findings. Cardiac enzymes revealed raised troponin $\mathrm{I}(\mathrm{TnI})$ at $1.81 \mathrm{ng} / \mathrm{mL}$ and creatine kinase-muscle/brain (CK-MB) was 13.9 $\mathrm{ng} / \mathrm{mL}$. Suspecting cardiac injury, a trans-thoracic echocardiography (TTE) was ordered which to our surprise revealed global hypokinesia with an ejection fraction of $25 \%$. To rule out any large vessel and coronary injuries, the patient underwent CT aortogram and CT coronary angiogram, both of which were normal studies. During the stay in the ED, the patient had repeated SVT and ventricular tachycardia (VT) which would resolve spontaneously. Serial TnI showed an increase to $6.19 \mathrm{ng} / \mathrm{mL}$. On the basis of clinical picture, ECG changes, abnormal TTE findings and rising trend of TnI, the patient was diagnosed with myocardial contusions and admitted under the cardiothoracic team in the intensive care unit. He was kept on conservative management in the ICU but had recurrent VT and unfortunately succumbed to his injuries after approximately 24 hours. The next of kin of the patient consented for the publication and the same has been archived.

\section{Discussion}

We present a case of myocardial contusions as described above. Since there is no definitive diagnostic tool, we came to a conclusion of myocardial contusions on the basis of arrhythmias in ECG, rising levels of cardiac troponins and left ventricular dysfunction in a previously normal heart.

Blunt cardiac injury can be graded into six subsets as described by Moore et al. [4] [Table 1]. Myocardial contusion is defined as a bruise of the cardiac muscle, the severity of which varies with the severity of injury and when the injury
Table 1: The American Association for the Surgery of Trauma classification for cardiac injuries as described by Moore et al. [4]

Grade I

1. Blunt cardiac injury with minor EKG abnormality (non specific ST of $\mathrm{T}$ wave changes, premature atrial or ventricular contractions, or persistent sinus tachycardia.

2. Blunt or penetrating pericardial wound without cardiac injury, tamponade, or cardiac herniation.

\section{Grade II}

1. Blunt cardiac injury with heart block or ischemic changes without cardiac failure.

2. Penetrating tangential cardiac wound, up to but not extending through endocardium, without tamponade.

Grade III

1. Blunt cardiac injury with sustained or multifocal ventricular contractions.

2. Blunt or penetrating cardiac injury with septal rupture, pulmonary or tricuspid incompetence, papillary muscle dysfunction, or distal coronary artery occlusion without cardiac failure.

3. Blunt pericardial laceration with cardiac herniation.

4. Blunt cardiac injury with cardiac failure.

5. Penetrating tangential myocardial wound, up to but not through endocardium, with tamponade.

Grade IV

1. Blunt or penetrating cardiac injury with septal rupture, pulmonary or tricuspid incompetence, papillary muscle dysfunction, or distal coronary artery occlusion producing cardiac failure.

2. Blunt or penetrating cardiac injury with aortic or mitral incompetence.

3. Blunt or penetrating cardiac injury of the right ventricle, right or left atrium.

\section{Grade V}

1. Blunt or penetrating cardiac injury with proximal coronary artery occlusion.

2. Blunt or penetrating left ventricular perforation.

3. Stellate injuries, less that $50 \%$ tissue loss of the right ventricle, right or left atrium.

\section{Grade VI}

1. Blunt avulsion of the heart.

2. Penetrating wound producing more than $50 \%$ tissue loss of a chamber. 
has occurred. It is one of the most uncommonly seen complications of blunt chest trauma [5]. Cardiac contusions present with a spectrum of clinical symptoms. The most severely injured patients may be hemodynamically unstable. In blunt poly-trauma patients, other obvious causes of hemodynamic instability like hemorrhagic shock, tension pneumothorax, tamponade, etc. may mask cardiac contusions.

Diagnosing myocardial contusions can be very difficult as patients have non-specific signs and symptoms. Workup for myocardial contusions includes ECG, cardiac biomarkers, echocardiography and nuclear imaging but none of them are $100 \%$ sensitive [6]. Table 2 enlists the recommendations to approach suspected myocardial contusions [7]. In suspected myocardial contusions, ECGs are the first step in diagnosis with a class 1 recommendation. Most patients with cardiac contusions will have evidence of arrhythmias in the first 24-48 hours. Co-relating rising troponin I levels with ECG changes helps in the diagnosis of myocardial contusions. TTE is a class II recommendation for the diagnosis of myocardial contusions and an abnormal study would make the diagnosis more likely [8]. International consensus on how to best diagnose myocardial contusions in blunt trauma patients still remains ambiguous. Depending on the criteria used for diagnosis, incidence rates have ranged from $8-76 \%$ [3].

Autopsy findings in BCI patients revealed almost $60-100 \%$ patients to have suffered from myocardial contusions [9]. Autopsy still remains the most conclusive method to establish the diagnosis. There have been reports where initially stable patients have subsequently succumbed to myocardial contusions [10]. Suspected patients with myocardial contusions need to be monitored and observed and require supportive therapy.

In our case, the patient was a young male with no cardiac history. After ruling out all the
Table 2: Diagnosing myocardial contusions as described by The Eastern Association for the Surgery of Trauma [7].

Level I Recommendation

1. Admission EKG should be obtained in all patients where there is suspected BCI.

Level II Recommendation

1. If admission EKG is abnormal, the patient should be admitted for continuous EKG monitoring for 24-48 hours. If admission EKG is normal, further pursuit of diagnosis should be abandoned.

2. If the patient is hemodynamically unstable, an imaging study such as TTE or TEE should be obtained.

3. Nuclear medicine scans add little compared with echocardiography and are not useful if echocardiography has been performed.

Level III Recommendation

1. Elderly patients with known cardiac disease, unstable patients, and those with abnormal admission EKGs can be safely operated on provided that they are closely monitored.

2. The presence of a sternal fracture does not predict the presence of $\mathrm{BCI}$, and does not necessarily indicate that monitoring should be performed.

3. Neither CPK analysis nor measurement of circulating cardiac troponin $\mathrm{T}$ is useful in predicting which patients have or will have complication related to BCI.

other causes of shock, co-relating clinical picture with abnormal ECGs, rising cardiac enzymes and abnormal TTE, the diagnosis of myocardial contusions was made. Unfortunately our patient did not survive. We conclude that it is imperative for emergency physicians to suspect cardiac contusions in the workup of stable and unstable blunt thoracic injuries.

\section{Conclusion}

We recommend emergency physicians to have a high index of suspicion for traumatic myocardial contusions and correlate clinical scenario with ECGs, enzymes and TTE to diagnose the same. If left undiagnosed, patients may spiral towards unwanted mortality. 
Contributors: SJ: manuscript writing and patient management; AV, AKK, WRS: manuscript editing, and patient management. AV will act as a study guarantor. All authors approved the final version of the manuscript and are responsible for all aspects of the study.

Funding: None; Competing interests: None stated.

\section{References}

1. Verma A, Kole T. International normalized ratio as a predictor of mortality in trauma patients in India. World J Emerg Med. 2014;5:192-195.

2. Hanschen M, Kanz K-G, Kirchhoff C, Khalil PN, Wierer $\mathrm{M}$, van Griensven $\mathrm{M}$, et al. Blunt cardiac injury in the severely injured - A retrospective multicentre study. PLoS One. 2015;10(7): e0131362.

3. Feghali NT, Prisant LM. Blunt myocardial injury. Chest. 1995;108:1673-1677.

4. Sybrandy KC, Cramer MJM, Burgersdijk C. Diagnosing cardiac contusion: old wisdom and new insights. Heart. 2003;89:485-489.
5. Moore EE, Malangoni MA, Cogbill TH, Shackford SR, Champion HR, Jurkovich GJ, et al. Organ injury scaling. IV: Thoracic vascular, lung, cardiac, and diaphragm. J Trauma. 1994;36:299-300.

6. Bertinchant JP, Polge A, Mohty D, Nguyen-Ngoc-Lam $\mathrm{R}$, Estorc J, Cohendy R, et al. Evaluation of incidence, clinical significance, and prognostic value of circulating cardiac troponin I and T elevation in hemodynamically stable patients with suspected myocardial contusion after blunt chest trauma. J Trauma. 2000;48:924-931.

7. Pasquale MD, Nagy K, Clarke J. Practice management guidelines for screening of blunt cardiac injury. J Trauma. 2012;73:S301-S306.

8. Alborzi Z, Zangouri V, Paydar S, Ghahramani Z, Shafa M, Ziaeian B, et al. Diagnosing myocardial contusion after blunt chest trauma. J Tehran Heart Cent. 2016;11:49-54.

9. Schultz JM, Trunkey DD. Blunt cardiac injury. Crit Care Clin. 2004;20:57-70. 